\title{
Intelligence: Beyond a monolithic concept
}

\author{
GEORGE E. MARSH II and ASGHAR IRAN-NEJAD \\ University of Alabama, Tuscaloosa, Alabama
}

\begin{abstract}
The relationship of intelligence to biology has been ignored because the nervous system has been considered too difficult to comprehend. In this article we argue that the sources of human intelligence are biological in nature and are inherited from ancestral species. Segregated from biology, the concept of intelligence has inherent limitations as a monolithic construct. Viewed from a biological perspective, intelligence is the capacity of the central nervous system for selfregulation under external (or stimulus-regulated), dynamic (or microsystem-regulated), and mindful (or person-regulated) control.
\end{abstract}

Current uses of the term "intelligence" are reminiscent of Wittgenstein's (1953) account of the concept of game. Intelligence is used interchangeably with competence, expertise, native talent, cultural talent, personal control, schooling, social interaction, and, of course, IQ. What is common to these various applications of the term "intelligence'? Only the spelling of the word itself. In the late 1970 s, a similar problem haunted the literature on the notion of "schema," which was defined as the building block of cognition (Rumelhart, 1980). Perhaps there are inherent limitations in the systematic use of monolithic concepts that otherwise serve us well in informal daily communication (Iran-Nejad \& Ortony, 1984).

Also problematic are definitions of monolithic constructs. For instance, Gardener (1986) defined intelligence as "an ability (or skill) to solve problems or to fashion products which are valued within one or more cultural settings"' (p. 74). According to this definition, scientists such as Copernicus, Galileo, and Pasteur were not intelligent because their solutions and products encountered ridicule and scorn.

In this paper, we assume that there are three core elements of the concept of intelligence: (1) biology, (2) problem solving, and (3) survival. All other aspects of the concept of intelligence (e.g., pedagogical, social, cultural, motivational, cognitive aspects) must be explained in terms of these core elements. Early approaches to the study of intelligence kept biology at a distance because the role of the nervous system is difficult to specify, a situation that we have addressed elsewhere (Iran-Nejad, Marsh, \& Clements, in press). We believe that biology must be directly considered in the attempt to define intelligence, because the nervous system is innately biological, and it is the only known system capable of producing natural intelligence. Problem solving and survival, on the other hand, have historically been important components of theories of intelligence. Within the biological, evolutionary perspective, the sources of human intelligence are found in previous

Correspondence should be addressed to George Marsh, College of Education, University of Alabama, Tuscaloosa, AL 35487-0231. ancestral species and in the legacy of nervous systems as they gradually evolved into the human brain, which is suitable for the aspects of human intelligence that never existed before in any other species.

\section{Intelligence and Perception}

Experimental psychology pioneered research on intelligence within the domain of perception. In keeping with this historical tradition, we will focus on the important problem of perceptual intelligence. As a product of evolution, the nervous system must have first overcome problems of survival at the perceptual level.

Imagine a study in which the subjects must solve a perceptual problem posed with sentences such as, $A B C D$ is the front of a cube and $E F G H$ is the front of a cube. They are asked to respond "yes" or "no" in reference to the Necker cube drawing shown in Figure 1. Then, during a subsequent testing phase, they are asked to participate in a recognition test of (1) the cube on the left, (2) the cube on the right, and (3) both cubes. To perform this task successfully, the nervous system must solve several problems associated with the (1) immediate, (2) ongoing, and (3) recurrent perceptions of the Necker cube stimulus and its two interpretations.

Emergent intelligence. As far as immediate stimulus perception is concerned, the simplest assumption is that a set of functionally independent microsystems in the visual subsystem fire under the direct influence of a corresponding set of independent stimulus elements. A coincidental stimulus constellation (CSC), like one of those shown in Figure 2, can cause coincidental momentary constellation firing (CMCF) in the visual subsystem of the brain. This is a purely emergent solution; but it goes a long way toward solving the problem of immediate perception (emergent intelligence). Its main advantage is that it requires no explicit pattern detection devices (Rumelhart, 1977), no template matching (Neisser, 1967), and no preexisting schemes (Rumelhart, 1980), all of which require unnecessarily complex processing schemas (IranNejad \& Ortony, 1984).

It is equally important, however, to note how emergent intelligence resulting from CMCF falls short of a com- 

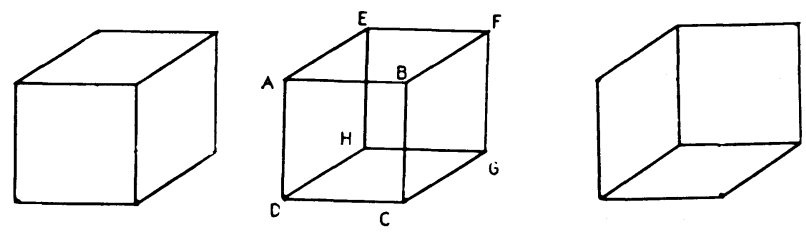

Figure 1. The Necker cube drawing and its interpretations.

prehensive solution to the problem of immediate perception. For instance, our immediate perception CMCFs for Figure 2a are more like the physical stimuli depicted in row $c$ than those in rows $a$ or $b$. In fact, the biofunctional model implies that the visual subsystem must create the same CMCFs for all three sets of CSCs in Figure 2, as well as for an indeterminate number of other CSCs-large, small, crude, precise, black and white, colored, and so on. Just how the brain accomplishes this feat has long been sought in pattern perception research. One answer may have to do with the density of the microsystems in the visual subsystem. The internal CMCFs for row a in Figure 2 are richer than their corresponding CSCs, because more microsystems participate in perception than there are elements in the available stimulus. This may be true, but it is unlikely to be the whole story.

Dynamic intelligence. Another aspect of the solution may have to do with what brain microsystems do when they participate in immediate perception. The biofunctional model implies that, having fired, each CMCF microsystem shifts from the initial firing to a different kind of activity to regulate its own functioning according to (1) the ongoing internal context and (2) dynamic principles of self-organization similar to those proposed by Gestalt psychology (dynamic intelligence). No preexisting network or set of connection weights is necessary.

With the emergence of dynamic intelligence, internal microsystem constellation activity becomes systematic rather than coincidental, and ongoing rather than momentary. Systematic ongoing brain activity (SOBA) differs from CMCF in that microsystems that participate in SOBA are all related to one another by virtue of an ongoing context (not by element-to-element connections)they constitute a nonconnectionist system (Iran-Nejad,

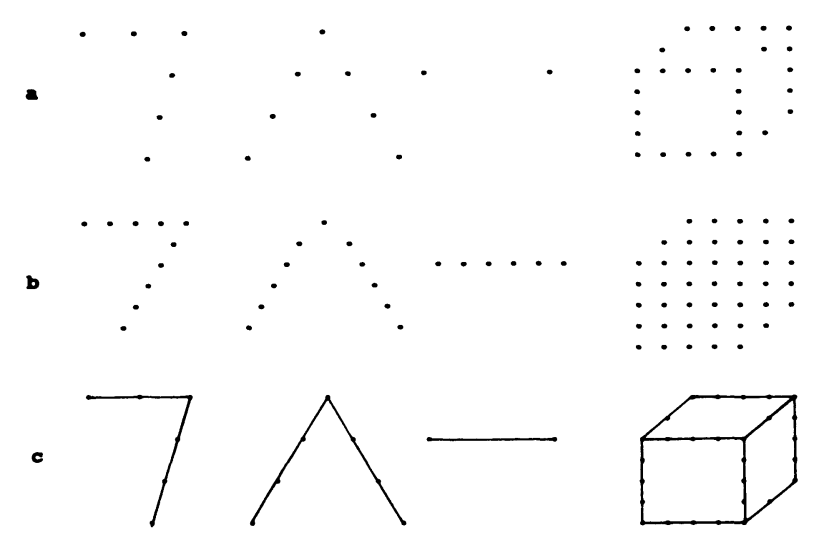

Figure 2. Coincidental stimulus constellations for coincidental momentary constellation firing in the visual subsystem.
1989). There is no such interrelationship among the microsystems participating in CMCF; here, coincidental firing is the only common characteristic. Thus, dynamic intelligence puts SOBA out of the realm of stimulus dependence and into the realm of ongoing brain functioning and makes it longer lasting, enabling the subject participating in our Necker cube study to have this perception available when not looking directly at the drawing.

Mindful intelligence. Ongoing perception also forms the basis for recurrent perception. But in order for recurrent perception to occur, the CSC must return to the visual field. If the brain were capable of practicing no more than emergent and dynamic intelligence, recurrent perception would be wholly dependent on external factors. With an abundance of Necker cube drawings in nature, recurrence would happen at least as often as would be necessary for survival. Alternatively, the individual must actively place the Necker cube drawing into the visual field again in the same way as it was originally perceived. The brain's solution to this kind of problem is mindful intelligence, which enables the individual to consciously establish and carry out the goal of placing the Necker cube drawing in the visual field. With emergent, dynamic, and mindful intelligence, the individual can successfully complete the Necker cube experiment.

\section{Intelligence as Self-Regulation}

If the present analysis is correct, what makes the nervous system "intelligent" is its capacity for self-regulation under external (or stimulus-regulated), dynamic (or microsystem-regulated), and mindful (or person-regulated) control (Iran-Nejad, 1990). Behaviorism, as well as neoconnectionism, assumes that organisms function under the influence of external control. Information processing theory implies that human functioning is active and mindful. Neither school can fully explain the performance of subjects in the Necker cube experiment. For instance, mindfully focusing attention on ABCD to determine if it is a cube front does not guarantee that the two-dimensional drawing is perceived as a three-dimensional cube. For most viewers, the third dimension moves into focus unwittingly-or under the influence of dynamic self-regulation.

The contribution of dynamic self-regulation can be illustrated in yet another way. An intriguing aspect of the two cubes embedded in the Necker cube drawing is that they are available to mindful perception only one at a time. Yet the individual perceives not two discrete objects, but one of two interrelated cubes each time. It is difficult to postulate how the perceptual system can assemble this interrelationship by means of mindful intellectual activity. This consideration, and the fact that most viewers report that the flip-flop from one cube to another occurs by itself without being fully under mindful control, suggests that dynamic intelligence plays the major role in establishing this relationship.

\section{Intelligence in Its Broader Context}

The concept of intelligence is often reserved for humans, with other organisms being excluded as unintelligent or simply controlled by stereotyped or predetermined processes. 
But if human intelligence is the product of evolution, it is legitimate to ask the following question: Other than differences in the ability to solve abstract problems, do other organisms and humans share similar intellectual features? This question may be more revealing if answered from an evolutionary perspective. Organisms have become what they are today because the systems of which they are built overcame fundamental survival problems during evolution.

Coincidental assortments of molecules formed into cells; collections of cells amassed into multicellular organisms; and increasingly complex organisms encountered and solved more complex survival problems at each ascending level, while still retaining the processes inherited from simpler organisms. The brain has its genesis in human progenitors, so the precursors of human intelligence must still be active phylogenetically, and humans and animals must share ancient genes. From this perspective, humans and other animals must share intellectual characteristics, even though in its fullest implication the concept of intelligence fits only humans.

Complexity is a natural consequence of evolution. Many animals on the ocean floor and on land are not any different from their fossilized ancestors at the dawn of time, whereas more complex life forms, including homo sapiens, are relative newcomers. But later evolved organisms retain certain characteristics of distant ancestors. Evolution does not reinvent complexity; rather, complexity is achieved with each species as a nonlinear dynamic of biological mechanisms. In addition to biostatic adaptations favoring selection in an ecological system, such as camouflage and built-in physical defenses like shells or claws, adaptation may have been possible in less physically endowed organisms because of different kinds of intelligence, each of which may have developed consecutively as organisms became more complex and as adaptive behavior became specialized within particular econiches.

In emergent intelligence, parts work together (by coincidence) to create something new and/or randomly solve a survival problem. Coincidental factors in the behavior of organisms emerged to favor selection; while many life forms died out, others succeeded because of coincidental circumstance. A compelling example of emergent intelligence is the sea urchin, which feeds on the sand dollar. The problem facing the sea urchin is to consume its prey before it becomes buried in the safety of the sand. To do so, sea urchins go beneath the sand dollar at different parts of its perimeter and appear to prevent it from burrowing. An observer might be impressed at the apparent teamwork, perhaps believing that sea urchins have defined roles and agree to cooperate. However, emergent intelligence might explain how sea urchins can survive in an environment by coincidence. A plentiful supply of sea urchins arrives, and they happen to suspend enough of a plentiful supply of sand dollars.

The precursors of emergent and dynamic intelligence are also evident in plant survival and growth. Plant seeds clearly depend on a coincidental constellation of environmental factors to grow, which fits broadly within the concept of emergent intelligence. The processes become dy- namic when interaction occurs among various parts within the physical system of the plant itself. It might be said that the system found a way to self-regulate because the parts can work together and regulate their own activities. Comparing the dynamic self-regulation involved in tree growth to skyscrapers reveals a significant difference, in that skyscrapers do not grow by themselves in nature because their parts are totally static and dependent on mindful intelligence, not of themselves but of humans. A tree, however, grows because its parts interact dynamically, although there is a great difference between the dynamic self-organization of trees and that of other organisms.

A similar case may be made for the organismic systems that serve as subsystems in larger systems. An excellent example is the immune system's solutions to illnesses. It is possible to explain the working of this system as a process of interacting emergent and dynamic intelligence. So too is the blood circulation system's solutions as a relational medium among various body subsystems, including the all-spreading environment for the immune system and hormones (Iran-Nejad \& Ortony, 1984).

Emergent and dynamic self-regulation may also be the mechanism by means of which evolution progressed to the social behavior of insects. A most remarkable example is evident in African termite mounds. The queen and her mate live in a self-imposed prison well below ground, where, in perpetual darkness, the queen is able not only to lay thousands of eggs but also to produce the appropriate kinds of eggs to replace soldiers and workers to restore an essential balance for the community. It is presumed that workers, who attend and feed the queen, also carry chemical information to her obtained from contacts among organisms higher in the mound who handle the food, providing both sustenance and the code for which kinds and quantities of eggs to produce in order to restore the proper balance of workers and soldiers in the colony. This is certainly a dynamic process, in which parts operate systematically in the context of the overall system. This same structure has probably been manifested in other biological systems.

In dynamic intelligence, the parts function together in a coordinated fashion appropriate for the ongoing context of the system as a whole. The parts form a coherent system to work toward solving a problem. Because knowledge is created dynamically, any change in one part has implications for the functioning of other parts. In a dynamic process, every combination of the parts is a new combination. Even combinations that repeat the past exactly are new combinations, in that the process replicates again.

It is within the nervous system, however, that emergent, dynamic, and mindful intelligence all come together into one system. Mindful intelligence, the most distinctive feature of humans, enables the species to reproduce activities of the past for future survival through planning, teaching, learning, and recorded knowledge. This type of intelligence, although relatively new in geologic time, is undoubtedly rooted in emergent and dynamic intelligence. The origin of mindful control was made possible because of the evolutionary development of active self-regulation to control brain processes. This model permits theoretical con- 
sideration of the human brain for intellectual activity in addition to intentional learning strategies and analytical thinking. This concept of intelligence includes processes that have evolved into human intelligence. An exclusive focus on the uniquely human characteristics of intelligence (i.e., analytical reasoning, symbolic information processing) obscures the fact that other stages of development were critical and are still innately important for fostering intelligence. The brain is a large interactional system that engages in nonlinear dynamic activities as well as in intentional problem solving.

\section{DISCUSSION}

Throughout history, in virtually all fields and disciplines, opposing theories have existed simultaneously. In science, progress has come from conflict between opposing theories. For example, during the first period of Greek philosophy, from which Western science derives much of its original inspiration, the early Ionian thinkers explained the philosophical problem of "being" in terms of combinations of natural elements. Later Greek thinkers approached it differently, objecting to such concepts of concrete substances and adopting logic and theoretical constructs in their explanations. Atomic theory emerged out of this debate (Durant, 1966).

Over the last few decades, similar circumstances have existed, as behaviorists, geneticists, and cognitive psychologists have taken different views about the brain and intelligence. But few have questioned the basic assumptions. In fact, there have been few attempts to define intelligence beyond the constructs of particular studies or tests.

\section{Different Views of Intelligence}

The chief ways of viewing intelligence are derived from work in experimental psychology, genetics (e.g., Piaget), and statistical analyses. Actually, theories of intelligence have grown out of very deliberate efforts to measure differences among people for practical purposes. Binet and his collaborator Simon did this to separate slow-learning school children. Galton, Bertillion, and others had earlier devised tests, including measures of sensory thresholds, grip strength, cranial and facial measurements, and analysis of fingerprints, to identify the feebleminded and criminals. Many now famous persons formed the list of test developers and intelligence theorists, including Thorndike, Thurstone, Spearman, Cattell, Terman, Wechsler, and, most recently, Kauffman. Only after the testing tradition was well established did some scientists turn to the task of defining intelligence, something that some practical test developers have thought unnecessary, apparently accepting Boring's (1923) operational definition that "intelligence as a measurable capacity must at the start be defined as the capacity to do well in an intelligence test.' Although this comment has been used both as a criticism and as a practical justification of intelligence tests, it was used by Guilford (1967) as the cornerstone for his famous text, The Nature of Intelligence, in which he took inspiration from Boring to argue that intelligence can be revealed through the statistical technique of multivariate analysis. Earlier, Spearman asserted that intelligence was not a unitary factor, and Thurstone applied factor analysis of existing tests and derived five primary mental abilities; later, Guilford elaborated on the statistical model to achieve a structure of the intellect, containing, in three dimensions, 120 cells embracing all possible intellectual elements.

Cognitive psychologists, ignoring the statistical traditions, use the computer as the model for research on cognition and intelligence in two general approaches: artificial intelligence (computationalism) and artificial neural networks (connectionism), based on parallel processing. Both of these approaches disregard the biological basis of the brain or the nature of problems and survival needs that created natural intelligence. Cognitive psychologists try to model how problems are solved, either in symbolic or subsymbolic ways. "Expert" programs are used by computationalists to manipulate signs said to represent human thinking or "intelligent" human tasks, such as playing chess. Connectionists use programs to imitate the neural architecture of the brain. The computer is said to imitate neurons connected through axons and dendrites, and these are said to reveal highly complex mathematical formulas.
Scientists have traditionally focused on differences between humans and other animals and ignored their similarities, particularly with regard to intelligence, tool use, and language. But while scientists reluctantly admit to similarities, many eagerly accept claims that computer programs can think just as humans do. As Pagels (1988) noted:

In fact, they don't care about the brain at all. This simulation problem, however, is not well defined because concepts and symbols are not well defined outside of their context, which is the entire world. It is not at all clear how or if one can program a computer to do such a simulation. (p. 307)

\section{The Utility of a Three-Core Conceptualization of Intelligence}

To disregard the biological nature of the brain itself and accept statistical, computer, and mathematical models leads further into complex representations of intelligence that may not relate to anything in the natural world. As a product of mammalian phylogenetic development, the human brain contains ancient vestiges in common with other species. If the apex of human evolutionary development is abstract intelligence served by language, it is misleading to focus only on mindful intelligence, as if it were but a mere appendage to the rest of the organism.

By distributing intelligence as emergent, dynamic, and mindful, the brain is capable of performing higher level functions because not everything must be controlled by a central executive. Thus, the three core elements of intelligence emerge as evolutionary processes endemic to the current intellectual capacities of homo sapiens. Understanding the nature of evolution-tested brain processes may lead to a better understanding of intelligence (Iran-Nejad \& Ortony, 1984).

We conclude from our analysis above as follows: (1) All aspects of the concept of intelligence must be explained in terms of biology, problem solving, and survival; (2) many aspects of the central nervous system are self-regulatory; and (3) computer and information processing models and simulations of intelligence are restricted to the specific products of mindful intelligence and represent mathematical rather than biological processing.

\section{REFERENCES}

Boring, E. G. (1923). Intelligence as the tests test it. New Republic. 34, 35-37.

Durant, W. (1966). The life of Greece. New York: Simon \& Schuster. GARDENER, H. (1986). The waning of intelligence tests. In R. J. Sternberg \& D. K. Detterman (Eds.), What is intelligence? Contemporary viewpoints on its nature and definition (pp. 73-76). Norwood, NJ: Ablex.

GuILFORD, J. P. (1967). The nature of intelligence. New York: McGrawHill.

IRAN-NeJAD, A. (1989). A nonconnectionist schema theory of understanding surprise-ending stories. Discourse Processes, 12, 127-148.

IrAN-NejAD, A. (1990). Active and dynamic self-regulation of learning processes. Review of Educational Research, 60, 573-602.

Iran-Nejad, A., Marsh, G. E., II, \& Clements, A. C. (in press). The figure and the ground of constructive brain functioning: Beyond explicit memory processes. Educational Psychologist, 27.

IrAN-Nejad, A., \& ORTONY, A. (1984). A biofunctional model of distributed mental content, mental structures, awareness, and attention. Journal of Mind \& Behavior, 5, 173-210.

NeISSER, U. (1967). Cognitive psychology. New York: Appleton.

Pagels, H. R. (1988). The dreams of reason: The computer and the rise of the sciences of complexity. New York: Bantam Books.

Rumelhart, D. E. (1977). Toward an interactive model of reading. In S. Dornic (Ed.), Attention and performance (Vol. 4, pp. 573-603). Hillsdale, NJ: Erlbaum.

Rumelhart, D. E. (1980). Schemata: The building blocks of cognition. In R. J. Spiro, B. C. Bruce, \& W. F. Brewer (Eds.), Theoretical issues in reading comprehension: Perspectives from cognitive psychology, linguistics, artificial intelligence, and education (pp. 33-58). Hillsdale, NJ: Erlbaum.

Wittgenstein, L. (1953). Philosophical investigations. New York: Macmillan. 